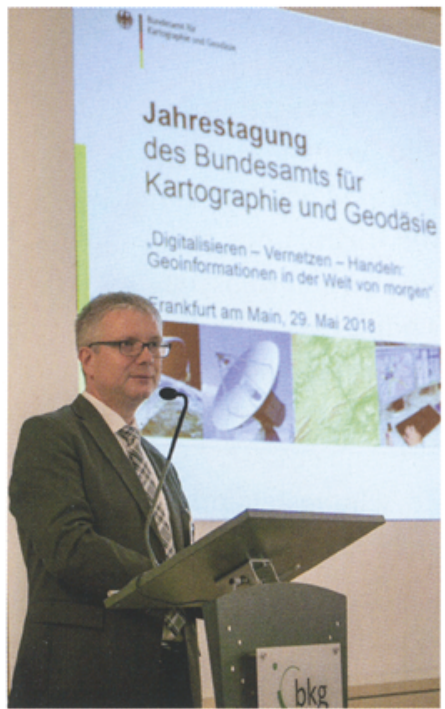

Der Präsident des BKG, Hansjörg Kutterer, eröffnet die Jahrestagung

litik, Wirtschaft, Wissenschaft und Verwaltung. Für die digitale Gesellschaft sind verortete Informationen (Geoinformationen) ein entscheidender Faktor. Sie sind die Grundlage für strategisches Planen, fundiertes Entscheiden und politisches Handeln und stellen eine elementare Voraussetzung für Innovationsprozesse in Wirtschaft, Wissenschaft und öffentlicher Verwaltung dar.

Zum Veranstaltungsauftakt begrüßte der Präsident des BKG, Prof. Dr. Hansjörg Kutterer, die geladenen Gäste: „Geoinformationen in der Welt von morgen sind durchgängig digital. Sie erschließen unterschiedlichste The- men und ermöglichen neuartige Arbeitsprozesse und Geschäftsmodelle. Nutzungshindernisse wie komplizierte Lizenzsysteme sind beseitigt.“ Anschließend verlas Dr. Michael Frehse, Leiter der Abteilung Heimat im Bundesministerium des Innern, für Bau und Heimat (BMI), ein Grußwort und stellte dabei essenzielle Fragen, die es gilt, vor dem Hintergrund der Digitalisierung neu zu beantworten: „Wie können Informationen gewonnen, effektiv gesammelt und analysiert werden? Wie kann es gelingen, die wertvollen Informationen effizient für uns nutzbar zu machen? Wie sind die Informationen zu schützen?"

Dr. Rolf Densing, ESA-Direktor für Missionsbetrieb und Leiter des Raumfahrtkontrollzentrums (ESOC) in Darmstadt, präsentierte „Highlights und Herausforderungen des ESA-Satellitenbetriebs“. Auf „Wetterdaten als bedeutsame Geodaten im Kontext des digitalen Wandels" ging Prof. Dr. Gerhard Adrian, Präsident des Deutschen Wetterdienstes (DWD), ein. Mit Blick auf die stetig wachsenden Datenmengen und digitalen Prozesse betonte er: „Statt Daten zum Nutzer zu bringen, müssen wir den Nutzer zu den Daten bringen.“ $\mathrm{Zu}$ „Geodaten und Digitalisierung - Zwei wichtige Treiber in der Versicherungs- wirtschaft" führte Andreas Siebert, Leiter „Geospatial Solutions“ der Versicherungsgesellschaft Munich RE, aus.

Den Abschluss der Veranstaltung bildeten eine Diskussionsrunde zwischen den Rednern und dem Auditorium sowie weitere intensive Gespräche.

Eine begleitende Ausstellung verdeutlichte den Aufbau und die Aufgaben des BKG, das sich als Behörde im Geschäftsbereich des Bundesministeriums des Innern, für Bau und Heimat (BMI) mit der Beobachtung über die Datenhaltung bis hin zur Analyse, Kombination und Bereitstellung von Geodaten befasst. Von der Arbeit des BKG profitieren insbesondere Bundeseinrichtungen, die öffentliche Verwaltung, die Wirtschaft, die Wissenschaft - und fast jeder Bürger in Deutschland. Experten aus den verschiedensten Bereichen wie Verkehr, Katastrophenvorsorge, Innere Sicherheit, Energie und Umwelt verwenden Geodaten, Landkarten, Referenzsysteme und Informationsdienste des BKG für ihre Pläne und Untersuchungen. Das BKG unterhält ein Dienstleistungszentrum in Leipzig (www.geodatenzentrum. de) sowie geodätische Observatorien im In- und Ausland. Weitere Informationen finden Sie unter www.bkg.bund.de.

(nach Pressemitteilung)

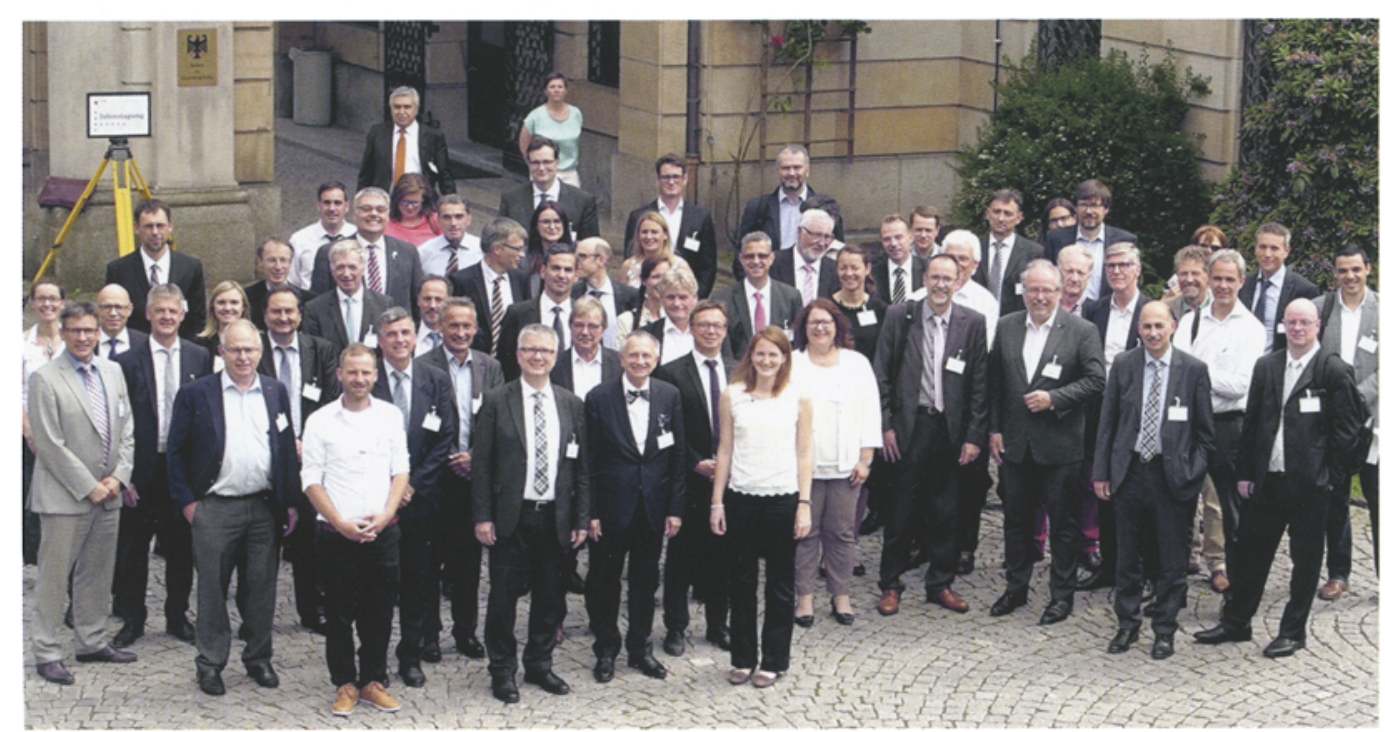

Die Teilnehmerinnen und Teilnehmer der Jahrestagung vor dem Eingang des Verwaltungsgebäudes des BKG

\section{Ruhr-Universität Bochum startet Kreativ-Wettbewerb zur Horizons-Mission}

Die Ruhr-Universität Bochum hat aus Anlass der Horizons-Mission des deutschen ESA-Astronauten Alexander Gerst Schülerinnen und Schüler zu einem Kreativwettbewerb aufgerufen unter dem Motto „Fliegt mit Alex ins All und setzt seinen Blick auf die Erde in künstlerischer Art und Weise um!"

Alexander Gerst ist am 6. Juni 2018 zur Internationalen Raumstation ISS aufgebrochen und wird dort ein halbes Jahr lang seine zweite Forschungsreise durchführen. Es ist üblich, dass ESA-Astronauten ihre Mission unter ein persönliches Motto stellen, für das Gerst den Namen „ISS Horizons“ gewählt hat. Der Missionsname „Horizons“ spiegelt die Neugier und Faszination wider, Unbekanntes in 400 Kilometern Höhe zu entdecken und zu erforschen. Gerst will mit der Mission neue Horizonte erreichen und überwinden. In „Horizons“ sieht er die Fortführung seiner „Blue Dot“-Mission.

Deutsche Universitäten, Forschungseinrichtungen, Firmen und das DLR beteiligen sich an der Mission mit zahlreichen Experimenten, die sich auf die Themenbereiche Biologie, Medizin, (Astro-)Physik und Materialwissenschaften beziehen. In diesem Rahmen findet ein Bildungsprogramm für Kinder und Jugendliche statt.

Der Wettbewerb trägt den Titel „self-eSTEAM“. Dieser Name setzt sich aus mehreren Begriffen zusammen: Selbstwertgefühl (self-esteem), „„“ für E-Learning, „STEAM“ für „science“, „technology“, „engineering“, „art“ und „mathematics“. Damit werden auch die wichtigsten Themenfelder des Wettbewerbs umrissen, der sich an Schulklassen, AGs oder Teams aus den Klassen 5 bis 10 aller Schulformen richtet. Näheres ist auf der Website http://www.ruhr-uni-bochum.de/ self-esteam/ zu erfahren. 